\title{
The antenatal diagnosis of placenta accreta
}

\author{
CH Comstock, ${ }^{a, b, c}$ RA Bronsteen ${ }^{a}$
}

a Department of Obstetrics and Gynecology, William Beaumont Hospital, Royal Oak, MI, USA ${ }^{\mathrm{b}}$ Department of Obstetrics and Gynecology, University of Michigan, Ann Arbor, MI, USA ${ }^{c}$ Department of Obstetrics and Gynecology, Wayne State University, Detroit, MI, USA Correspondence: CH Comstock, William Beaumont Hospital, Division of Fetal Imaging, 3601 West Thirteen Mile Road, Royal Oak, MI 48071, USA.Email chcomstock@comcast.net

Accepted 31 August 2013.

The incidence of placental attachment disorders continues to increase with rising caesarean section rates. Antenatal diagnosis helps in the planning of location, timing and staffing of delivery. In at-risk women grey-scale ultrasound is quite sensitive, although colour ultrasound is the most predictive. Magnetic resonance imaging can add information in some limited instances. Patients who have had a previous caesarean section could benefit from early (before 10 weeks) visualisation of the implantation site.
Current data refer only to placentas implanted in the lower anterior uterine segment, usually over a caesarean section scar.

Keywords Caesarean hysterectomy, caesarean section, colour Doppler ultrasound, magnetic resonance imaging, placenta accreta, placenta increta, placenta percreta, placenta, scar pregnancy, three-dimensional colour Doppler ultrasound, ultrasound.

Please cite this paper as: Comstock CH, Bronsteen RA. The antenatal diagnosis of placenta accreta. BJOG 2014;121:171-182.

\section{Introduction}

Risk factors for placental attachment disorders (PAD) have been clearly documented-placenta praevia, previous caesarean section and age are the most common. ${ }^{1,2}$ More infrequent risk factors are Asherman syndrome ${ }^{3}$ and endometrial ablation. ${ }^{4-6}$ An increase has also been seen in in vitro fertilisation pregnancies. ${ }^{7}$ Placenta accreta, increta and percreta (abbreviated as placental attachment disorders or $\mathrm{PAD}$ in the following discussion) account for 33-50\% of all emergency peripartum hysterectomies. ${ }^{8-10}$ Clearly this is a major healthcare problem.

The exact process by which PAD is initiated is still unknown but is probably more complicated than just abnormal decidualisation of a scarred area or lack of decidua in the lower uterine segment near the cervix. The consequences are often caesarean hysterectomy and the end of fertility, as well as increased rates of blood loss and transfusion, injury to surrounding organs, and increased rates of intensive care admission when compared with women who undergo caesarean section for placenta praevia alone. ${ }^{11}$ Forewarning and preparation, including a multidisciplinary approach, do help to reduce morbidity, ${ }^{12-14}$ but even with careful preparation morbidity can be considerable. ${ }^{11,15-17}$ Delivery at an institution with experience, operative resources, and access to large amounts of blood and blood replacement products is ideal because significant amounts of blood can be lost and it is not possible to predict which women will lose the most. ${ }^{16}$

The following discussion reviews the two major diagnostic modalities, ultrasound and magnetic resonance imaging (MRI), used at the present time to attempt a prenatal diagnosis. There has been a change in the past several years in the evidence for various techniques used to make a diagnosis.

\section{Methods}

A PUbMed search was made using the terms 'placenta accreta', 'diagnosis of placenta accreta', 'imaging of placenta accreta', 'magnetic resonance in placenta accreta', 'placenta percreta', 'colour Doppler ultrasound and placenta accreta', 'magnetic resonance imaging in placenta accreta','early diagnosis placenta accreta', and 'caesarean scar pregnancy'. In the evaluation of imaging signs only original papers published in English or translated into English were used. Pathological examination of the uterus was the preferred proof of an accreta because myometrial fibrils can normally be found on removed placentas and clinical descriptions by multiple clinicians regarding causes of bleeding and difficulty removing a placenta can be subjective. An emphasis was placed on recent papers because MRI resolution and colour Doppler continue to improve. 


\section{Ultrasound}

\section{First trimester}

We know that placenta accreta and percreta can occur in a first-trimester pregnancy from reports in which a dilatation and curettage was performed with subsequent massive bleeding. ${ }^{18-21}$ When the uterus was examined the placenta was found to be invasive or adherent with lack of decidua between the myometrium and placenta. It is reasonable to assume that abnormal placentation was present from the time of implantation. Although a few cases have been reported in women with no previous uterine surgery, the most frequent risk factor was previous caesarean section. In a small series of women who had proven PAD, four of whom had reached near term, retrospective review of all available first-trimester scans showed the gestational sac implanted low and anteriorly rather than in its normal fun-
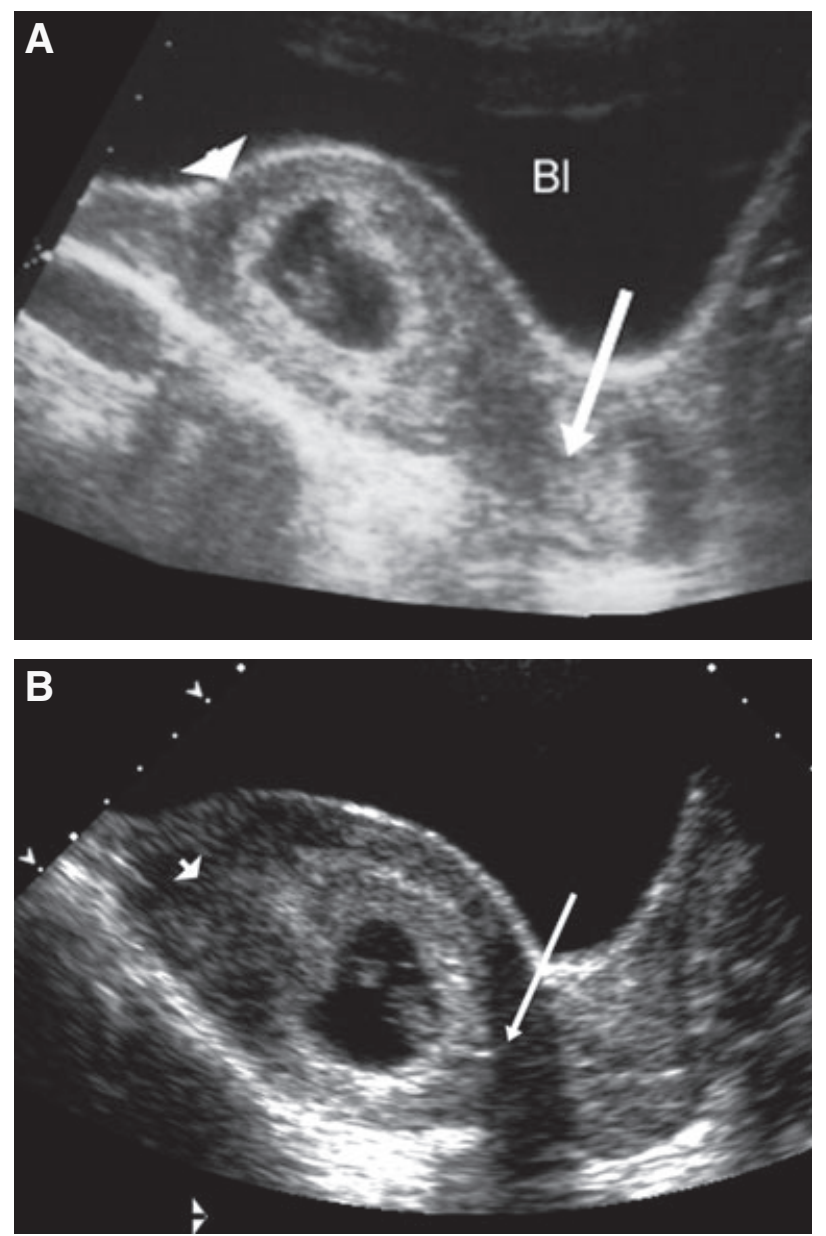

Figure 1. (A) Early pregnancy. Sagittal view. Normal implantation of a gestational sac in the fundus (arrowhead). Cervix near long arrow. (B) Low implanted sac in a pregnancy which went to term. Cervix and internal os near arrow. Note the thick anterior myometrium and the continuous white line representing the bladder-uterine wall interface.

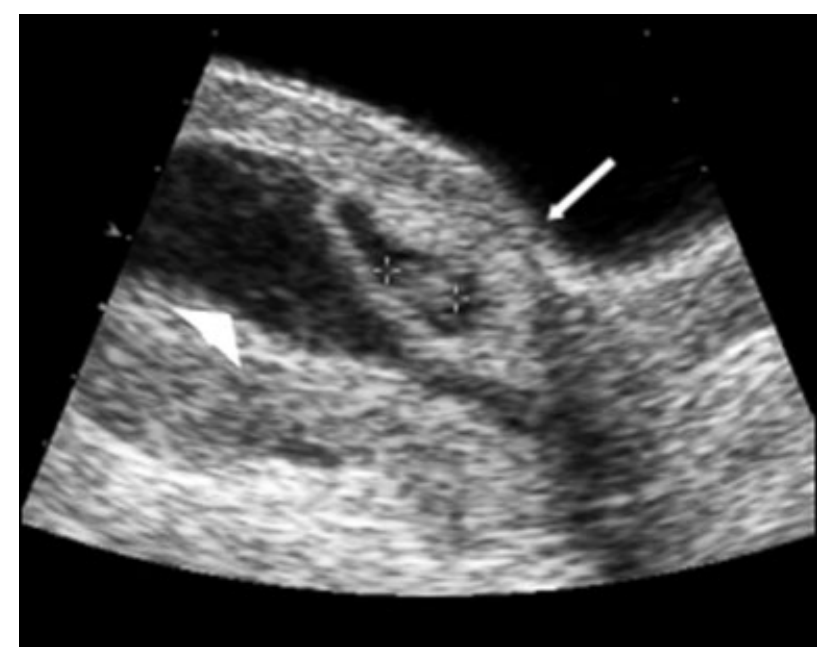

Figure 2. Low implanted pregnancy in placenta accreta. Sagittal view. The sac is implanted low and anteriorly on a caesarean section scar (long arrow). The anterior myometrium is very thin. Fluid outlines the confines of the endometrial cavity.

dal position (Figures 1 and 2) in all six women who had had previous caesarean sections. The anterior myometrium was thin but visible over the scar. In the one case in which the sac was fundal in location there had been no previous lower uterine scar. ${ }^{22}$ If the diagnosis had been made in the first trimester a recommendation would have been made to terminate the pregnancy because rupture of the uterus with ensuing maternal shock in the second and early third trimesters is a risk. This study was repeated by Ballas, but in a wider gestational age range of $8.5-14$ weeks. ${ }^{23}$ In the two early cases ( 8 weeks) the sacs were located in the lower uterine segment. The average gestational age in the other eight women was 13 weeks and, although it was stated that these gestational sacs were implanted low, it would be difficult to determine this in most cases. However, in eight of the ten women, including those at 8 weeks of gestation, echolucent areas were seen in the placentas, confirming a previous report of early lacunae, ${ }^{24}$ and nine had an irregular placenta-myometrium interface.

These low-lying sacs should be differentiated from the occasional sac found in the lower uterine segment associated with impending demise and subsequent passage, or even the occasional normal pregnancy, though this is quite rare (Figure 1B). Impending demise can be distinguished from both a normal but low implanted pregnancy and a pregnancy implanted on a scar by lack of surrounding blood flow and by movement within the cavity when pressure is exerted on the anterior uterus.

The overall incidence of these early accreta pregnancies is not known, but presumably if all women with previous caesarean sections who are later identified as having $\mathrm{PAD}$ were scanned before 10 weeks of gestation (when 
the sac does not occupy the entire uterine cavity) the gestational sac would be implanted in the anterior lower uterine segment.

Occasionally these cases of first-trimester PAD are termed 'cervicoisthmic' pregnancies, although that term implies growth of the placenta into the cervix as well as the myometrium. Although this term does not apply to every pregnancy attached to a caesarean scar, certainly in some there is extension into the cervix. Their course is similar however-they can proceed to term at which time attempted separation of the placenta results in massive haemorrhage. ${ }^{25}$

The literature and terminology are confusing because those pregnancies implanted low on a caesarean section scar are usually termed 'scar pregnancies', although that term strictly applies only to pregnancies that are actually within the scar surrounded by myometrium and therefore not within the endometrial cavity. ${ }^{26,27}$ In a retrospective review of hysterosalpingograms in 150 women with previous caesarean sections, $60 \%$ had defects at the site of the incision of which $65 \%$ were focal outpouchings and $35 \%$ were linear defects. ${ }^{28}$ True scar pregnancies lying completely within the myometrium would be a consequence of entry of the fertilised ovum into one of these linear sinus tracks within the scar. They can be responsible for a mass within the myometrium but outside the endometrial cavity and are obviously missed on dilatation and curettage. Continuing positive pregnancy tests and a suprapubic mass suggest the diagnosis. ${ }^{29}$ They have never been described as proceeding to the third trimester, unlike pregnancies implanted upon the scar (rather than within it). Confusion has also ensued because some authors have used the term 'scar pregnancy' for pregnancies implanted in the niche left from a previous caesarean section, but not surrounded by myometrium on all sides and therefore are not true 'scar pregnancies'. Because they lie within the divot left by a scar they can also be missed during early curettage, but presumably as they enlarge they grow out into the endometrial cavity. Recent review papers of 'scar pregnancies' actually appear to include a mixture of niche implantations and true scar pregnancies because some were managed by injection and then dilatation and curettage. ${ }^{30,31}$ For the latter to be successful, the pregnancy could not have been surrounded on all sides by myometrium. In either case the clinical management would be the same as reviewed recently. ${ }^{28,30,31}$

\section{Second and third trimesters}

Results can be divided into several parts: the performance of particular ultrasound signs, the overall accuracy of ultrasound in the diagnosis, the performance of MRI alone, and lastly the comparison of ultrasound to MRI. Ultrasound sensitivity depends on which imaging criteria are used.

Clear space. In many women with normal placentation a hypoechogenic space can be observed between the placenta and myometrium (Figure 3). However, McGahan et al. observed the clear space to be missing in a large percentage of normal pregnancies with anterior placentas but found it to be almost always present in women with a posterior placenta. $^{32}$ Finberg and Williams first noted that the absence of the retroplacental clear space was the one finding that accounted for almost all false positives in the diagnosis of PAD, ${ }^{33}$ a finding that has been confirmed by others. ${ }^{34,35}$ Wong et al. found absence of the clear space in 37 (65\%) women without placenta accreta and in $100 \%$ of those women with it. ${ }^{36}$ Hence, it is sensitive but not specific. The primary use of the clear space appears to be that its presence effectively excludes placenta accreta because it has a

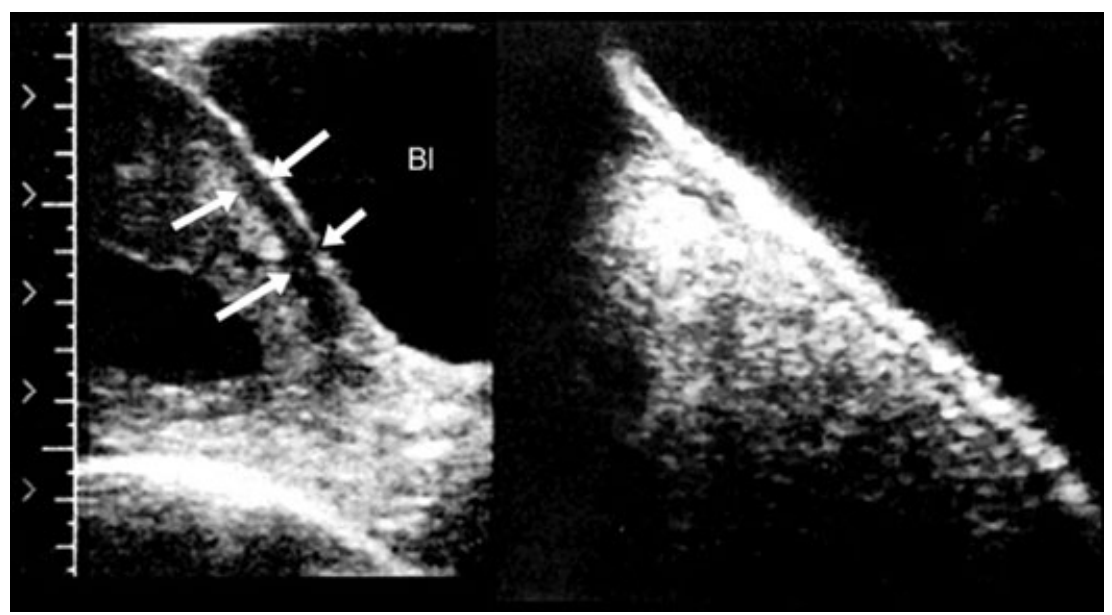

Figure 3. Sagittal view. Left, clear space (arrows) and right, absence of clear space in women with a normal pregnancy. Bl bladder. 
high negative predictive value (NPV). ${ }^{35,36}$ These findings are listed in Table 1.

Bladder line interruption. The interface between the uterus and bladder is represented on grey scale sonography as a continuous white line (Figure 3 ). Its loss can be relatively obvious (Figure 4) or more subtle, seen best on transvaginal ultrasound with a partially full bladder. In their large (41 women) pathology-proven series of women with previous caesarean sections and anterior placentas, Cali et al. confirmed Finberg and Williams findings that this is the sign

Table 1. Utility of the clear space in diagnosis of PAD

\begin{tabular}{lcccc}
\hline Author & $\begin{array}{c}\text { Sensitivity } \\
(\%)\end{array}$ & $\begin{array}{c}\text { Specificity } \\
(\%)\end{array}$ & PPV & NPV \\
\hline Comstock et al. $^{34}$ & 73 & & 14 & \\
Wong et al. $^{36}$ & 100 & 35 & 20 & 100 \\
Cali et al. $^{35}$ & 90.2 & 80.8 & 57 & 96.7 \\
\hline
\end{tabular}
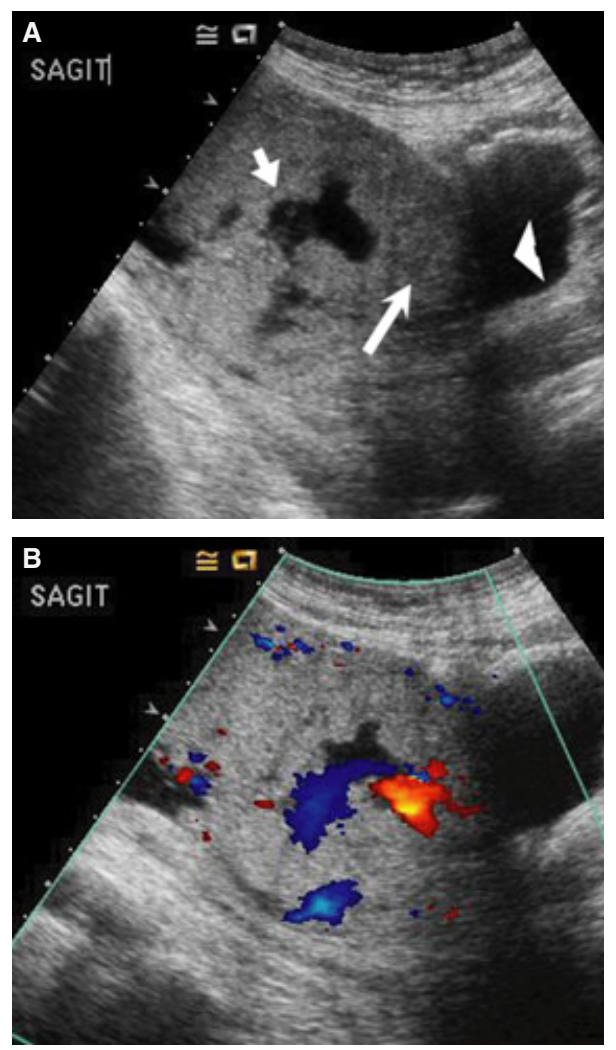

Figure 4. (A) Grey-scale sagittal view. Arrowhead shows loss of the usual white bladder-uterine serosal line. The large irregular space (medium arrow) is a lacuna and the mottled appearance near the large arrow represent very small ones. (B) Colour Doppler of the same area showing flow within the large lacunae. with the highest positive predictive value (PPV) for PAD. ${ }^{33,35}$ The interruption of this line is a result of increased vascularity in this space, as they showed using colour Doppler; it does not signify invasion of the bladder because interruption can be seen in placenta accreta. They showed that when this hypervascularity extends the entire width of the bladder-uterine interface placenta percreta is probable (see Colour Doppler section).

The cause of the low sensitivity reported in the papers by both Comstock et al. and Wong et al. may be that not all women had transvaginal ultrasound with the quite specific conditions used by Cali et al. ${ }^{35}$ In that large study the authors first determined that $300 \mathrm{ml}$ in the bladder resulted in the best visualisation of the uterine-bladder interface and then instilled this amount into each woman's bladder. Performance of bladder line interruption is summarised in Table 2.

Lacunae. The PPV of lacunae shows more variation from author to author than other signs of PAD. When observed by colour Doppler and spectral ultrasound, lacunae have high-velocity and low-resistance flow and are irregular in cross-section on grey-scale sonography ${ }^{35}$ (Figure 5). They are located deep in the placenta, not under the fetal surface of the placenta (Figure 6), and are irregularly shaped, not round as are placental lakes. They have been reported as

Table 2. Utility of interrupted bladder line in the diagnosis of PAD

\begin{tabular}{|c|c|c|c|c|}
\hline Study & $\begin{array}{c}\text { Sensitivity } \\
(\%)\end{array}$ & $\begin{array}{c}\text { Specificity } \\
(\%)\end{array}$ & PPV & NPV \\
\hline Cali et al. ${ }^{35}$ & 70 & 99 & 96 & 92 \\
\hline Comstock et al. ${ }^{34}$ & 20 & & 75 & \\
\hline Wong et al. ${ }^{36}$ & 11 & 100 & 100 & 88 \\
\hline
\end{tabular}

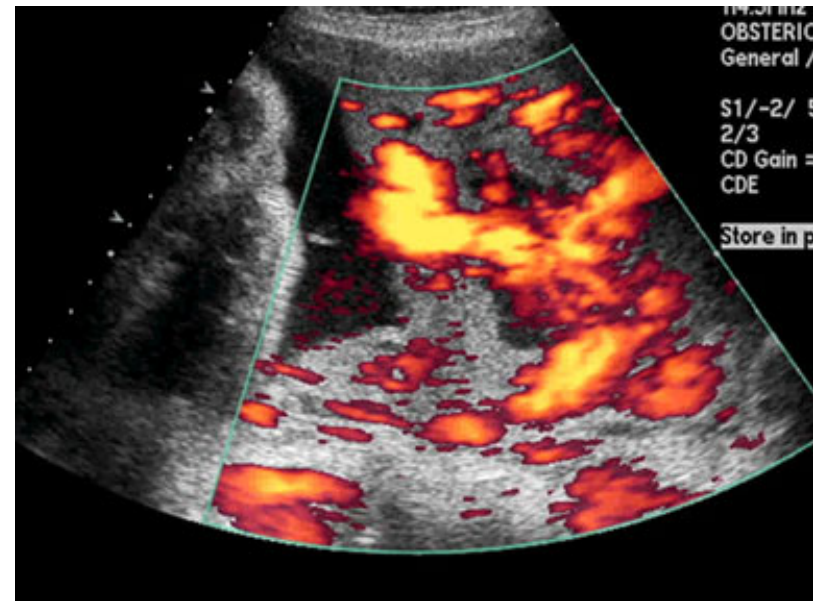

Figure 5. Placenta lacunae as seen with colour Doppler 


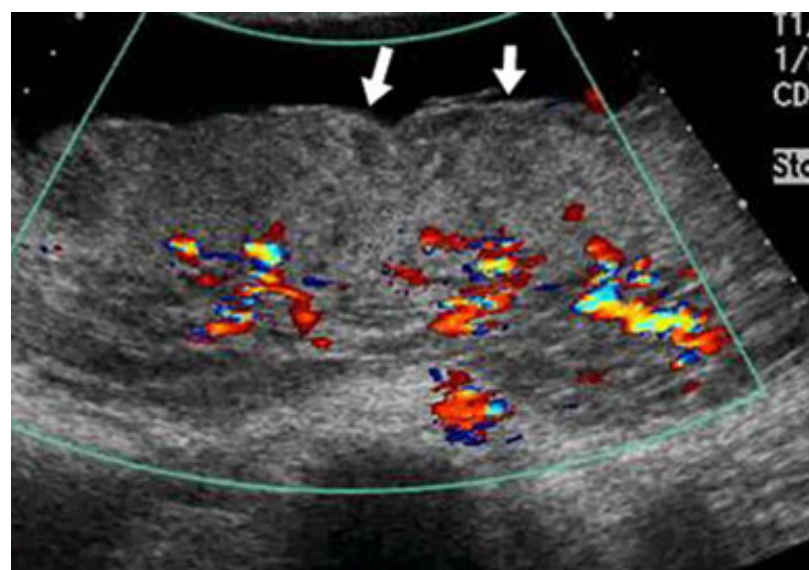

Figure 6. Dark spaces under the fetal surface of the placenta (arrows) should not be mistaken for lacunae.

sensitive and specific in some studies and not so in others. Comstock et al. found them to be $93 \%$ sensitive in women at 20 weeks of gestation and beyond with a $93 \%$ PPV. $^{34}$ The more lacunae the more likely there is placenta percreta. ${ }^{33}$ Using Finberg's scale of grade 0 when none were seen, $1+$ when one to three small lacunae were present, $2+$ when four to six larger or more irregular ones were seen and grade $3+$ when there were many throughout the placenta, Yang et al. also found that cases of normal placentation had grades 0 or 1 , in placenta accreta half had grade 0 and half had grade 1, and in increta or percreta only grades 2 and 3 occurred.$^{37}$ Grade 1 and above were associated with significantly increased rates of transfusion, disseminated intravascular coagulation, intensive care unit admission, and caesarean hysterectomy. Unfortunately $30 \%$ of women, those without caesarean hysterectomies, had evaluation of the placenta alone, which has been noted (above) to be unreliable. Despite the lack of pathology confirmation this study is included because it was done transvaginally and the results were divided into grades. The conclusion that can be drawn is that the more lacunae the more likely there will be a necessity of caesarean hysterectomy. This was confirmed by Chou et al. in a study with a high caesarean hysterectomy rate and therefore pathology confirmation. ${ }^{38}$ Cali et al. found that six or more lacunae were associated with placenta percreta in all 17 of their cases. ${ }^{35}$ Lacunae can appear as early as 8 weeks. ${ }^{24,25}$ The utility of lacunae in the diagnosis of PAD is summarised in Table 3.

Myometrial thickness. Myometrial thickness of less than $1 \mathrm{~mm}$ has been investigated by several authors. ${ }^{39,40}$ Its use is confounded by the fact that the myometrium of the lower uterine segment naturally thins as term is approached, but Wong et al. found a sensitivity of $22 \%$, specificity of $100 \%$, PPV of $100 \%$ and NPV of $89 \%$ (nine women) and Twickler et al. found a sensitivity $100 \%$, spec-
Table 3. Utility of lacunae in the diagnosis of PAD

\begin{tabular}{|c|c|c|c|c|}
\hline Study & $\begin{array}{c}\text { Sensitivity } \\
(\%)\end{array}$ & $\begin{array}{c}\text { Specificity } \\
\text { (\%) }\end{array}$ & PPV & NPV \\
\hline Cali et al. ${ }^{35}$ & 73 & 86 & 60 & 90 \\
\hline Comstock et al. ${ }^{34}$ & 93 & & 93 & \\
\hline Wong et al. ${ }^{36}$ & 100 & 28 & 21 & 100 \\
\hline Yang et al. ${ }^{37}$, Gr. $\geq 1$ & 86.9 & 78.6 & 76.9 & 88 \\
\hline Yang et al. ${ }^{34}$, Gr. $\geq 2$ & 100 & 97.2 & 93.8 & 100 \\
\hline
\end{tabular}

Gr 1 = grade 1 (one to three lacunae), Gr. 2 = grade 2 (four to six lacunae).

ificity $72 \%$, PPV $72 \%$, and NPV 100\% (nine women). Loss of the myometrial wall higher in the uterus can be ominous (Figure 7).

\section{Ultrasound-colour/colour power/colour power three-dimensional Doppler}

One of the best uses of colour Doppler is to investigate the presence or absence of flow within echolucent areas of the placenta: lacunae are very obviously high flow areas. However, if on grey scale the lacunae are irregularly shaped and within the substance of the placenta, then colour Doppler probably does not add information.

There is normally a clear demarcation of placenta from the uterus, even in the absence of a clear space, with the placental edge forming a well-defined arc. Wong et al. have written about 'tissue interface disruption' on grey scale in which this usual smooth interface between the uterus and placenta is disrupted (Figure 5). On colour Doppler, vessels can be seen bridging the placenta to myometrium. When the two findings were combined in a small series of nine women, sensitivity, specificity, PPV and NPV were 89,96 , 80 and $98 \%$, respectively.

Chou et al. found that high-flow vessels linking the placenta and bladder had a high sensitivity and specificity for $\mathrm{PAD}^{38}$ However, they warned that care must be taken to show that these vessels truly connect the placenta and bladder because two false positives were the result of bladder varices in women who had had previous caesarean sections. Its use in distinguishing placenta accreta from placenta percreta is discussed below.

Interruption of the uterine serosa bladder line is probably caused by hypervascularity. This appears to be easier to detect using colour Doppler than grey scale since Cali et al. noted a $70 \%$ sensitivity using grey scale but a $90 \%$ sensitivity using colour Doppler. ${ }^{35}$

Placenta accreta is not necessarily more benign than placenta increta or percreta ${ }^{17}$-large amounts of blood can be lost in either case. However, several authors have attempted to distinguish accreta from increta in the antenatal period. Using three-dimensional colour Doppler both Cali et al. 

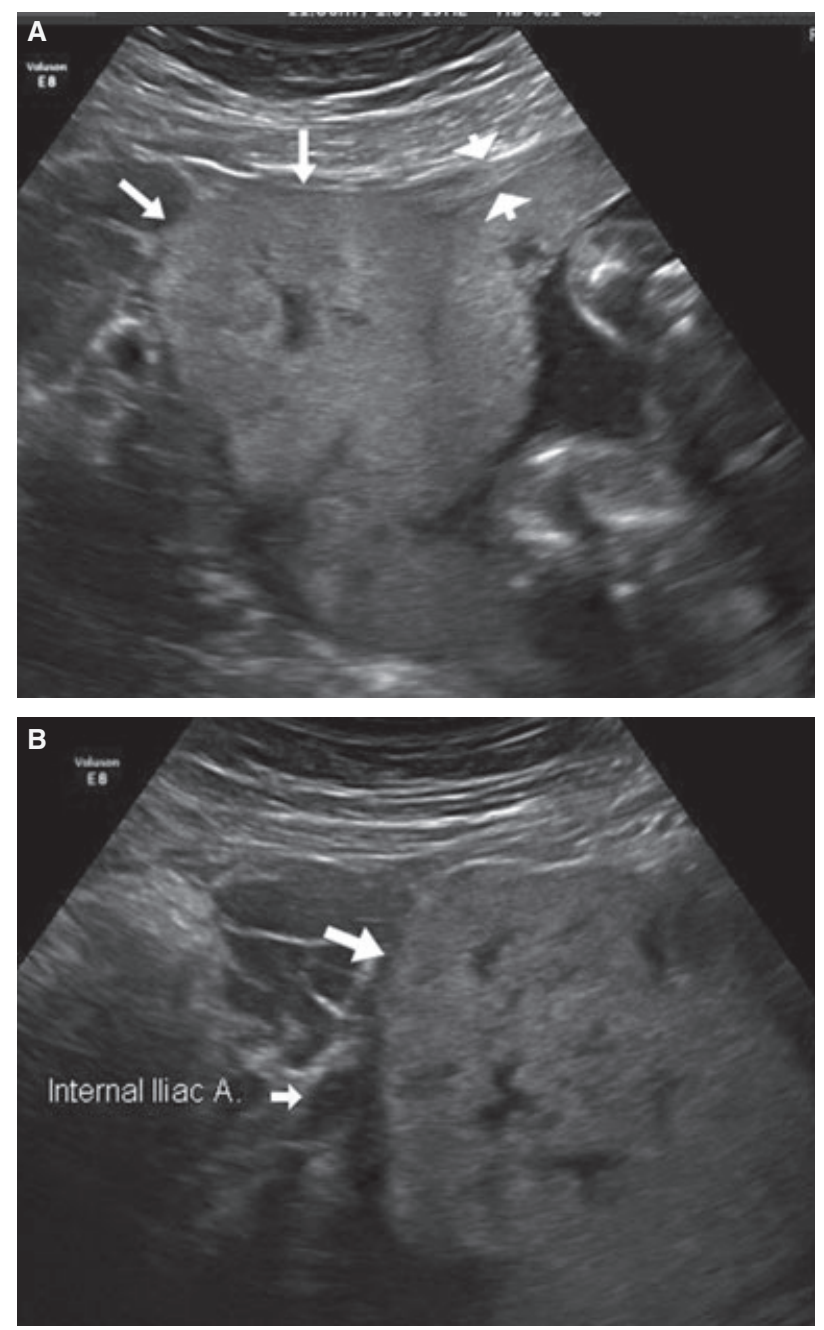

Figure 7. (A) Placenta percreta into the broad ligament. The uterine wall (arrowheads) disappears in the area of the arrows. (B) No uterine wall is seen between the placenta and the veins of the broad ligament. There are large lacunae within the placenta.

and Chalubinski et al. ${ }^{41}$ found that extension of large vessels over the width of the placenta, from left to right, correlated with placenta percreta. Using three-dimensional colour power Doppler Cali et al. demonstrated that the increased vascularity seen at the uterine bladder interface extended from side to side (as seen on the coronal view) in all 17 cases of placenta percreta with a sensitivity, specificity, NPV and PPV of 90, 100, 100 and 97\%, respectively (Figure 8). In the latter, placenta accreta/normal placenta was able to be separated from placenta increta/percreta with $100 \%$ sensitivity, specificity, PPV and NPV using this method. ${ }^{41}$

\section{Combination of ultrasound (grey scale and colour Doppler)} signs

The accuracy of diagnosis appears to improve using more than one ultrasound finding. Using three grey-scale
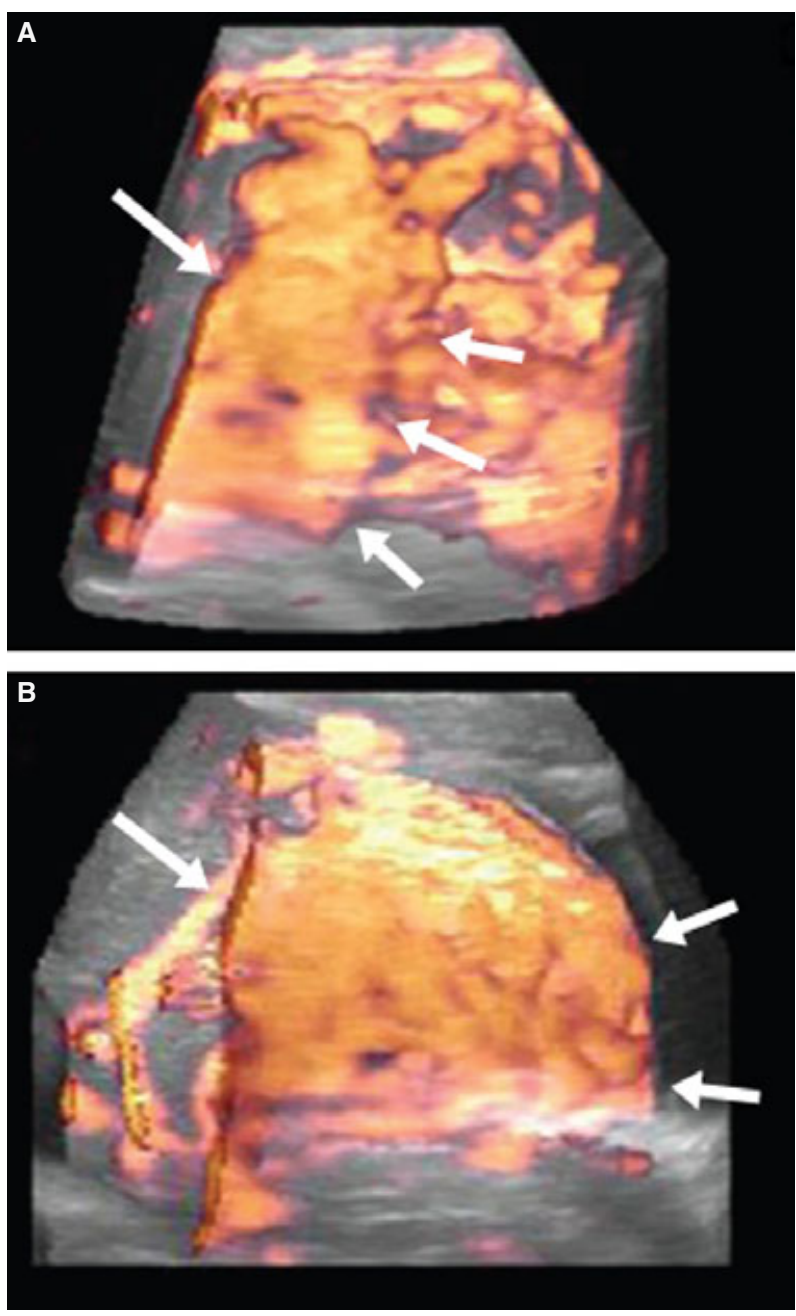

Figure 8. Three-dimensional power Doppler imaging, coronal view. (A) Serosa bladder wall interface neovascularisation (arrows) involving part of the bladder-uterine interface in placenta accreta and (B) involving all of the interface from side-to-side (arrows) in percreta. (Cali et al., with permission from U/trasound Obstet Gynecol).

signs (clear space, bladder line interruption, lacunae) and two three-dimensional colour Doppler signs (tortuous confluent vessels crossing placenta width and hypervascularity of uterine serosa bladder wall) Cali et al. found that all 41 women with PAD had two or more signs. Half of the women with four signs had placenta percreta and all of the women with five signs had placenta percreta. None of the women without PAD had more than one criteria. Comstock et al. used three grey-scale signs and found that when using two or more criteria the sensitivity was $80 \%$ and the PPV was $86 \%$. However, lacunae by themselves had a higher sensitivity of $93 \%$ and a PPV of $93 \% .^{34}$ Esakoff et al. found that grey scale ultrasound had an overall sensitivity of $89 \%$, specificity of $91 \%$, PPV of $68 \%$ and NPV of $97.6 \%$. However, no analysis was published of individual signs. ${ }^{11}$ This paper 
also differed from that of Cali et al. by including all women with placenta praevia whether or not they had had previous surgery.

\section{Magnetic resonance imaging}

There are three areas to be addressed when assessing MRI in the detection of PAD: the best MRI signs, the sensitivity and specificity compared to ultrasound, and the determination of when this more expensive test can contribute additional information. Unfortunately, the number of different study designs and the relatively low number of women make MRI difficult to evaluate. Confounding this is a variation of ultrasound criteria used for comparison and, in addition, using multiple interpreters. ${ }^{42}$ Almost all studies required a suspicious ultrasound examination before the woman was sent for MRI, which created an ascertainment bias and alerted the reader that the woman had an abnormal ultrasound examination. ${ }^{42-46}$ Additionally, some papers did not require pathology proof but relied on varying clinicians' opinions as to difficulty of removing the placenta ${ }^{42,46-48}$ or in most women relied on examination of the placenta alone for a histological diagnosis. ${ }^{42,46,48}$ Others involved more than one hospital and non-uniform MRI protocols. ${ }^{45}$

One paper involved the use of gadolinium as a second MRI test but did not separate the results of the first stage from the second. ${ }^{46}$ Gadolinium is known to cross the placenta and to be excreted by the fetal kidney. Since the effects on fetal growth and fetal kidneys are unknown it is not routinely used, although it certainly increases the contrast between the uterus and placenta. ${ }^{46}$ Many studies did not provide enough clinical information to judge in which women MRI would provide better information than ultrasound.

Lim et al., however, performed MRI on at-risk women regardless of positive or negative ultrasound findings, had pathological proof of PAD from caesarean hysterectomies and included considerable clinical information. ${ }^{49}$ The MRI was interpreted by one experienced person. They found that the volume of dark placental bands (first described by Lax) was the most predictive finding in true PAD (Figure 9). These bands were also seen in women without PAD but in those they were quite small in volume. Whether these bands represent haemorrhage or infarct or fibrosis is not clear and the authors warn that it is possible that these could be found in cases of placental infarction from other causes such as intrauterine growth restriction. In the one case that was a false positive on both ultrasound and MRI the ultrasound diagnosis was made using absence of the clear space, the most common cause of a false-positive ultrasound. The MRI finding was a large band in a woman found to have a large placental infarct but with no PAD. Not enough data were presented to determine the sensitiv-
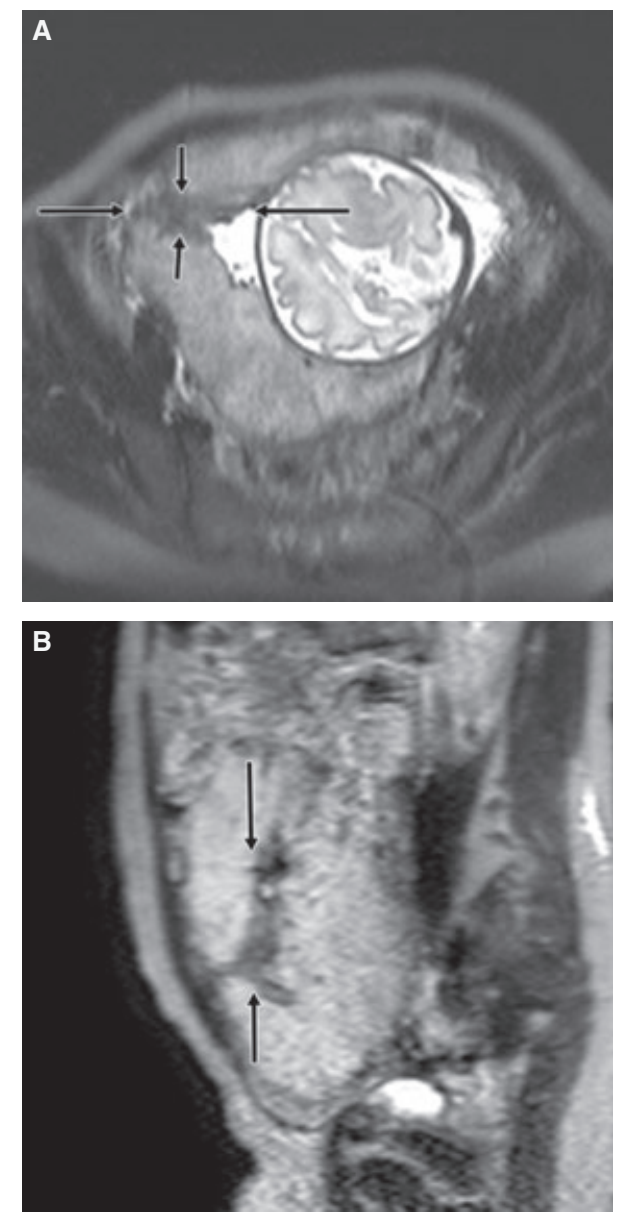

Figure 9. MRI of dark bands. (A) Dark arrows point to dark band measurements in the cross-sectional view (B) and in the sagittal view to calculate the volume of the band. (Lim et al., with permission from $A J R)$.

ity and specificity and NPV and PPV of ultrasound versus MRI. Although the authors state that MRI was more sensitive, the difference was one case. Lacunae were found in the ultrasound examinations of all true positives and no true negatives. Unfortunately, ultrasound criteria did not include evaluation of the uterine-bladder wall line or require transvaginal ultrasound. Two cases had false-negative ultrasound and MRI - both were accretas with blood loss of just $1000 \mathrm{ml}$. In these two cases of false-negative ultrasound and MRI and in one additional one in which ultrasound was negative but MRI was positive the placentas were lateral or posterior, not implanted over the caesarean section scar. In all three cases blood loss was minimal and the diagnosis was accreta. Could the lack of findings be related to their location away from the scar?

Derman et al. confirmed others' findings that the most reliable sign is the larger dark band on T2 Haste series. ${ }^{44}$ They added an additional finding - vessels of $6 \mathrm{~mm}$ or greater (which presumably correspond to lacunae). 
Alamo et al. found that the sensitivity and specificity of MRI depended upon both the type of attachment disorder and also the experience of the observer. ${ }^{45}$ In a study of 25 women with possible PAD on ultrasound, 12 were proven to have either accreta, increta or percreta by pathology examination of a removed uterus. Experienced readers detected PAD with $90 \%$ sensitivity and a $75 \%$ specificity whereas junior readers were $81 \%$ sensitive and $75 \%$ specific (all blinded to outcome). Junior readers were as skilled at detecting the most distinguishing sign of PAD — dark intraplacental bands. However, in distinguishing placenta accreta and increta from placenta percreta, senior readers had a sensitivity of $92 \%$ and specificity of $90 \%$ versus 76 and $88 \%$, respectively, for junior readers. Hence, senior readers were particularly good at detecting infiltration of adjacent organs by evaluating tenting of the bladder, interruption of the myometrial line and direct infiltration of pelvic organs. Of course the number of placentas percreta was small (five), as in most studies. Overall they found T2 hypointense placental bands, a focally interrupted myometrial border, infiltration of the pelvic organs and tenting of the bladder to be the best signs. Bulging of the uterus was not helpful because this can be seen in women with negative results. The sagittal view was much preferred, as other investigators have mentioned, and fast imaging with steady-state precession and single fast spin $\mathrm{t} 2 \mathrm{w}$ sequences were the most useful.

All studies of MRI versus ultrasound are underpowered. Dwyer et al. calculate that 194 women would need to have both ultrasound and MRI in a paired study design to have an $80 \%$ power to detect a difference at the $P=0.05$ level, and even more women would be needed in an unpaired study design. ${ }^{43}$ In their study no significant difference between the two modalities could be shown. They did find them to be complementary - when sonography was inconclusive MRI provided the correct diagnosis in four of five women and when MRI was inconclusive sonography provided the correct diagnosis in seven of eight women. They found that the ability of either to correctly diagnosis PAD was not affected by history of uterine surgery or placental location.

\section{Discussion}

As the number of cases has increased, awareness of the possibilities and importance of antenatal diagnosis has resulted in more attempts to identify reliable diagnostic signs. Colour Doppler ultrasound, and particularly the use of three-dimensional colour Doppler, has made visualisation of vessels much easier. MRI has advanced in acquisition times and thinness of slices. The major papers with good pathological correlation have been reviewed here. The strongest of these is the paper by Cali et al.-with 41 pathology-proven cases and use of both colour and three-dimensional Doppler.
Findings can be seen as early as a gestational sac can be identified if the sac is implanted in the anterior lower uterine segment. In the second and third trimesters findings may be seen as early as 18 weeks. ${ }^{34}$ Possible errors include using the absence of the clear space alone to make the diagnosis and confusing lacunae with placental lakes or subchorionic fluid collections and failing to evaluate the uterine bladder line with transvaginal ultrasound.

Colour Doppler in the hands of experienced people can add information and, when available in three dimensions, can distinguish accreta from percreta in most cases. The utility of this effort though is controversial because placenta accreta, the most benign of the three possibilities, can result in as much bleeding as a placenta percreta. Both require delivery at institutions able to transfuse large quantities of blood. Although new surgical approaches have been proposed, such as hypogastric artery balloons, these have not been definitively shown to reduce blood loss and may not be available. Ureteral stents often, but not always, help in avoiding transection of the ureters. Still there may be extensive growth into the broad ligament, bowel or bladder requiring some additional surgical skills either from the primary surgeon or consultant that are more likely to be available in a larger hospital.

Although the incidence of placenta accreta is on the rise, most institutions still see few cases, making the power of most studies inadequate. To overcome this, some investigators have gathered cases from several institutions. Unfortunately, these are almost always retrospective and not organised so that the same ultrasound signs and interpreters are used across locations. In addition, most interpreters are not blinded. In many publications reports of difficulty removing a placenta are used rather than proof from pathological evaluation of the uterus. Although this method may identify mild placenta accreta, many observers are involved and it is hard to interpret difficulty in removing the placenta or causes of bleeding. In other publications examination of the placenta is used to make the diagnosis but it is known that myofibrils on a placenta do not make a diagnosis of placenta accreta-they can be found on placentas with uneventful deliveries. Additional limitations are found in papers comparing ultrasound and MRI because usually the woman had to have had a suspicious or inconclusive ultrasound examination to be referred, so introducing ascertainment bias. Often the ultrasound examination in those studies was from a referring institution and was not re-evaluated by the group performing the MRI study. Most limitations were mentioned in the results.

The questions remain as to when and what MRI can contribute. If an ultrasound examination is thought to be positive there is little to be gained from MRI. However, if the findings suggest possible percreta or are inconclusive or negative in an at-risk woman MRI can be useful. Invasion 
of adjacent organs can be seen better on MRI than on ultrasound. Situations in which MRI may also contribute additional information include women with placenta praevia with a posterior or lateral implantation, a posterior scar from a myomectomy, a history of difficult placental removal in the past with a posterior or lateral placenta in the present pregnancy, or a history of endometrial ablation

Women with placental implantation not over a scar include women who have had a caesarean section but have implantation and accreta posteriorly and women who have never had uterine surgery. There is very little data about women who have not had uterine surgery because in most papers this is a requirement for inclusion. Interestingly in the above paper on reader experience only three of the 12 with PAD had had a previous caesarean section although 11 or the 12 had a placenta praevia. ${ }^{45}$ Hence this may be one of the few papers that addresses placenta accreta in the absence of previous uterine surgery. No comments were made as to the location of the placenta in the women or any differences in the findings between those who had had previous surgery and those who had not. We do know that placenta praevia alone is a risk factor for PAD and in the paper by Lim et al. there was one woman out of nine without previous surgery. In that case there were typical findings on both ultrasound and MRI for PAD. The ultrasound examination showed a lateral praevia with lacunae and the MRI showed a moderate-size dark band. Chou et al. notes that in all three women with false-negative colour Doppler results the placentas were posterior. Levine et al. noted one false-negative ultrasound diagnosis in a posterior placenta in their series. ${ }^{50}$ Hence there is scant information about the ultrasound or MRI findings in women who have placenta praevia but have had no previous uterine surgery or in whom the placenta is not implanted over a caesarean section scar.

Should women who have had a caesarean section be screened for placenta accreta early in pregnancy? A prospective screening study divided 105 at-risk women from 11 to 14 weeks of gestation into high-risk and low-risk categories depending on whether or not the placenta was over the identified uterine scar as seen on transvaginal ultrasound. ${ }^{51}$ Among the six women in the high-risk group just one had PAD at term. Interestingly, that woman was the only one who had an anterior low-lying placenta when scanned again early in the second trimester. No mention was made about lacunae. Available retrospective studies suggest that if the woman is at high risk (multiple caesarean sections, previous difficulty removing the placenta, endometrial ablation) an early scan before 10 weeks, rather than at 11-14 weeks, could be very useful if it shows a low implanted sac with a thin anterior uterine wall. In the interests of the mother's health and future reproduction, this pregnancy could then be interrupted because it will probably lead to placenta accreta, or worse, uterine rupture from a percreta. Conversely, a normally implanted sac in the upper half of the uterus would make PAD highly unlikely.

Occasionally a woman will not have any risk factors or any imaging findings and still have PAD with severe haemorrhage, and even death, even at large and well-staffed hospitals, but it is the hope that prenatal imaging will reduce this previously not uncommon occurrence.

\section{Conclusions}

The consequences of an undetected placenta accreta can be severe, so antenatal detection is ideal. The first and most important step is to maintain a high index of suspicion: women should be asked about previous uterine surgery, endometrial ablation or assisted reproduction both during initial prenatal visits and at the time of ultrasound imaging. It is important that facilities without colour Doppler or MRI facilities should still attempt screening. Fortunately the most readily available modality, grey-scale ultrasound, has a good sensitivity and PPV (although colour Doppler is the most sensitive). In the absence of colour Doppler, or in addition to it, examination of the bladder-uterine interface for interruption with a partially filled bladder and transvaginal ultrasound appears to be the most useful sign. Placental lacunae are the next most useful and the absence of the clear space, although sensitive, is very non-specific and a source of false positives. MRI has not proved as useful as initially hoped but may provide additional information in women who are at risk but do not have anterior placentas or in whom the ultrasound findings are difficult to interpret. It is important to remember that the ultrasound findings described in the literature have to this point almost always only described implantation anteriorly in the lower uterine segment of women with placenta praevia and a previous caesarean section. No papers have addressed imaging in cases of accreta elsewhere such as the fundus.

\section{Disclosure of interests}

The authors have no interests to disclose.

\section{Contributions to authorship}

$\mathrm{CHC}$ conceived the idea, analysed data and wrote this paper. RAB analysed data and wrote this paper.

\section{Details of ethics approval}

None was needed since this is a review.

\section{Funding}

No funding was received.

\section{Acknowledgements}

The authors wish to thank Robert Lorenz MD for his review of and suggestions for the manuscript. 


\section{References}

1 Miller DA, Chollet JA, Goodwin TM. Clinical risk factors for placenta previa-placenta accreta. Am J Obstet Gynecol 1997;177:210-4.

2 Silver RM, Landon MB, Rouse DJ, Leveno KJ, Spong CY, Thom EA, et al. Maternal morbidity associated with multiple repeat cesarean deliveries. Obstet Gynecol 2006;107:1226-32.

3 Jewelewicz R, Khalaf S, Neuwirth RS, Vande Wiele RL. Obstetric complications after treatment of intrauterine synecthiae (Asherman's syndrome). Obstet Gynecol 1976;47:701-5.

4 Hare AA, Olah KS. Pregnancy following endometrial ablation: a review article. J Obstet Gynaecol 2005;25:108-14.

5 Hamar BD, Wolff EF, Kodaman PH, Marcovici I. Premature rupture of membrane, placenta increta, and hysterectomy in a pregnancy following endometrial ablation. J Perinatol 2006;26:135-7.

6 Herath RP, Singh N, Oligbo N. Antenatal uterine rupture in a pregnancy following microwave endometrial ablation. J Obstet Gynaecol 2011;31:82-3.

7 Esh-Broder E, Ariel I, Abas-Bashir N, Bdolah Y, Celnikier DH. Placenta accreta is associated with IVF pregnancies: a retrospective chart review. BJOG 2011;118:1084-9.

8 Habek D, Becarevic R. Emergency peripartum hysterectomy in a tertiary obstetric center: 8 year evaluation. Fetal Diagn Ther 2007;2:139-42.

9 Rahman J, Al-Ali M, Qutub HO, Al-Suleiman SS, Al-Jama FE, Rahman MS. Emergency obstetric hysterectomy in a university hospital: a 25-year review. J Obstet Gynaecol 2008;28:69-72.

10 Glaze S, Ekwalanga P, Roberts G, Lange I, Birch C, Rosengarten A, et al. Peripartum hysterectomy: 1999-2006. Obstet Gynecol 2008;111:732-8.

11 Esakoff TF, Sparks TN, Kaimal AJ, Kim LH, Feldstein VA, Goldstein $R B$, et al. Diagnosis and morbidity of placenta accreta. Ultrasound Obstet Gynecol 2011;37:324-7.

12 Eller AG, Bennett MA, Sharshiner M, Masheter C, Soisson AP, Dodson $\mathrm{M}$, et al. Maternal morbidity in cases of placenta accreta managed by a multidisciplinary care team compared with standard obstetric care. Obstet Gynecol 2011;117:331-7.

13 Warshak CR, Ramos GA, Eskander R, Benirschke K, Saenz CC, Kelly $T F$, et al. Effect of predelivery diagnosis in 99 consecutive cases of placenta accreta. Obstet Gynecol 2010;115:65-9.

14 Tikkanen M, Paavonen J, Loukovaara M, Stefanovic V. Antenatal diagnosis of placenta accreta leads to reduced blood loss. Acta Obstet Gynecol Scand 2011;90:1140-6.

15 Hoffman MS, Karlnoski RA, Mangar D, Whiteman VE, Zweibel BR, Lockhart $J$, et al. Morbidity associated with nonemergent hysterectomy for placenta accreta. Am J Obstet Gynecol 2010;202:628. e1-5.

16 Wright JD, Shai P, Herzog TJ, Shah M, Bonanno C, Lewin SN, et al. Predictors of massive blood loss in women with placenta accreta. Am J Obstet Gynecol 2011;205:38. e1-6.

17 Harden MA, Walters MD, Valente PT. Postabortal hemorrhage due to placenta increta: a case report. Obstet Gynecol 1990;75: 523-6.

18 Woolcott RJ, Nicholl M, Gibson JS. A case of placenta percreta presenting in the first trimester of pregnancy. Aust N Z J Obstet Gynaecol 1987;27:258-60.

19 Ecker JL, Sorem KA, Soodak L, Roberts DJ, Safon LE, Osthanondh R. Placenta accreta complicating a first trimester abortion. a case report. J Reprod Med 1992;27:893-5.

20 Walter AJ, McCullough AE, Patel MD, Cornella JL. Placenta increta presenting as delayed postabortal hemorrhage. Obstet Gynecol 1999;93:846

21 Gherman RB, McBrayer S, Tichenor J, Wing DA. Placenta increta complicating first-trimester D\&C. Obstet Gynecol 1999;93:845.
22 Comstock CH, Lee W, Vettraino IM, Bronsteen RA. The early sonographic appearance of placenta accreta. J Ultrasound Med 2003;22:19-23.

23 Ballas J, Pretorius D, Hull AD, Resnik R, Ramos GA. Identifying sonographic markers for placenta accreta in the first trimester. J Ultrasound Med 2012;31:1835-41.

24 Chen YJ, Wang PH, Liu WM, Lai CR, Shu LP, Hung JH. Placenta accreta diagnosed at 9 weeks' gestation. Ultrasound Obstet Gynecol 2002;19:620-2.

25 Avery DM, Wells MA, Harper DM. Cervico-isthmic corporeal pregnancy with delivery at term: a review of the literature with a case report. Obstet Gynecol Surv 2009;64:335-44.

26 Sadeghi H, Rutherford T, Rackow BW, Campbell KH, Duzyj CM, Guess MK, et al. Cesarean scar ectopic pregnancy: case series and review of the literature. Am J Perinatol 2010;27:111-20.

27 Rotas MA, Haberman S, Levgur M. Cesarean scar ectopic pregnancies. Obstet Gynecol 2006;107:1373-81.

28 Surapanini K, Silberzweig JE. Cesarean section scar diverticulum: appearance on hysterosalpingography. AJR Am J Roentgenol 2008;190:870-4

$29 \mathrm{Wu}$ W, Kim SC. Placenta increta after first trimester dilatation and curettage manifesting as an unusual mass: magnetic resonance findings. Acta Radiol 2007;48:938-40.

30 Jurkovic D, Hillaby K, Woelfer B, Lawrence A, Salim R, Elson CJ. First-trimester diagnosis and management of pregnancies implanted into the lower uterine segment Cesarean section scar. Ultrasound Obstet Gynecol 2003;21:220-7.

31 Timor-Trisch IE, Monteagudo A, Santos R, Tsymbal T, Pineda G, Arslan AA. The diagnosis, treatment and follow-up of cesarean scar pregnancy. Am J Obstet Gynecol 2012;207:44. e1-13.

32 McGahan JP, Phillips HE, Reid MH. The anechoic retroplacental area: a pitfall in diagnosis of placental-endometrial abnormalities during pregnancy. Radiology 1980;134:475-8.

33 Finberg HJ, Williams JW. Placenta accreta: prospective sonographic diagnosis in patients with placenta previa and prior cesarean section. J Ultrasound Med 1992;11:333-43.

34 Comstock CH, Love JJ, Bronsteen RA, Lee W, Vettraino IM, Huang $R R$, et al. Sonographic detection of placenta accreta in the second and third trimesters of pregnancy. Am J Obstet Gynecol 2004;190: 1135-40.

35 Cali G, Giambanco L, Puccio G, Forlani F. Morbidly adherent placenta: evaluation of ultrasound diagnostic criteria and differentiation of placenta accreta from percreta. Ultrasound Obstet Gynecol 2013;41:406-12.

36 Wong HS, Cheung YK, Zucollo J, Tait J, Pringle KC. Evaluation of sonographic diagnostic criteria for placenta accreta. I Clin Ultrasound 2008;36:551-9.

37 Yang JI, Lim YK, Kim HS, Chang KH, Lee JP, Ryu HS. Sonographic findings of placental lacunae and the prediction of adherent placenta in women with placenta previa totalis and prior Cesarean section. Ultrasound Obstet Gynecol 2006;28:178-82.

38 Chou MM, Ho ESC, Lee YH. Prenatal diagnosis of placenta previa accreta by transabdominal color Doppler ultrasound. Ultrasound Obstet Gynecol 2000;15:28-35.

39 Twickler DM, Lucas MJ, Balis AB, Santos-Ramos R, Martin L, Malone $S$, et al. Color flow mapping for myometrial invasion in women with a prior cesarean delivery. J Matern Fetal Med 2000; 9:330-5.

40 Wong HS, Cheung YK, Strand L, Carryer P, Parker S, Tait J, et al. Specific sonographic features of placenta accreta: tissue interface disruption on gray-scale imaging and evidence of vessels crossing interface-disruption sites on Doppler imaging. Ultrasound Obstet Gynecol 2007;29:239-41. 
41 Chalubinski KM, Pils $S$, Klein $K$, Seemann R, Speiser $P$, Langer $M$, et al. Antenatal sonography can detect the degree of placental invasion. Ultrasound Obstet Gynecol 2013;42:518-24.

42 Jaraquemada JMP, Bruno $\mathrm{CH}$. Magnetic resonance imaging in 300 cases of placenta accreta: surgical correlation of new findings. Acta Obstet Gynecol Scand 2005;84:716-24.

43 Dwyer BK, Belogolovkin V, Tran L, Rao A, Carroll I, Barth R, et al. Prenatal diagnosis of placenta accreta: sonography or magnetic resonance imaging? J Ultrasound Med 2008;27:1275-81.

44 Derman AY, Nikac V, Haberman S, Zelenko N, Ophsa O, Flyer M. MRI of placenta accreta: a new imaging perspective. AJR Am J Roentgenol 2011;197:1514-21.

45 Alamo L, Anass A, Rey J, Denys A, Bongartz G, Terraz S, et al. Detection of suspected placental invasion by MRI: do the results depend on observer' experience? Eur J Radio/ 2013;82:e51-7.

46 Warshak CR, Eskander R, Hull AD, Scioscia AL, Mattrey RF, Benirschke $K$, et al. Accuracy of ultrasonography and magnetic resonance imaging in the diagnosis of placenta accreta. Obstet Gynecol 2006;108:573-81.

47 Teo TH, Law YM, Tay KH, Tan BS, Cheah FK. Use of magnetic resonance imaging in evaluation of placental invasion. Clin Radiol 2009;64:511-6.

48 Lax A, Prince MR, Mennitt KW, Schwebach JR, Budorick NE. The value of specific MRI features in the evaluation of suspected placental invasion. Magn Reson Imaging 2007;25:87-93.

49 Lim PS, Greenberg M, Edelson MI, Bell KA, Edmonds PR, Mackey AM. Utility of ultrasound and MRI in prenatal diagnosis of placenta accreta: a pilot study. AJR Am J Roentgenol 2011;197: 1506-13.

50 Levine D, Hulka CA, Ludmir J, Li W, Edelman RR. Placenta accreta: evaluation with color Doppler US, power Doppler US, and MR imaging. Radiology 1997;205:773-6.

51 Stirnemann JJ, Mousty E, Chalouhi G, Salomon LJ, Bernard JP, Ville Y. Screening for placenta accreta at 11-14 weeks of gestation. Am J Obstet Gynecol 2011;205:547. e1-6.

\section{Commentary 'All women with prior caesarean section should have a 6- to 8-week scan to predict placenta accreta'}

The systematic review by Comstock et al., published in this issue, gives us confidence that an early ultrasound scan is sensitive and specific for the detection of placenta accreta. Appraisal of current scientific knowledge about placenta accreta will lead to the belief that we are in a cul-de-sac with this serious condition. There is an abundance of knowledge on the association of scar pregnancy and abnormal adherence of the placenta (Fylstra Ob Gynecol Survey 2002 57:537-543; Timor-Trisch et al. AJOG 2012;207:44.e1-13), This clinical link remains under-utilised. We think that it provides a clue to the solution of the problem of placenta accreta and suggests a hypothesis.

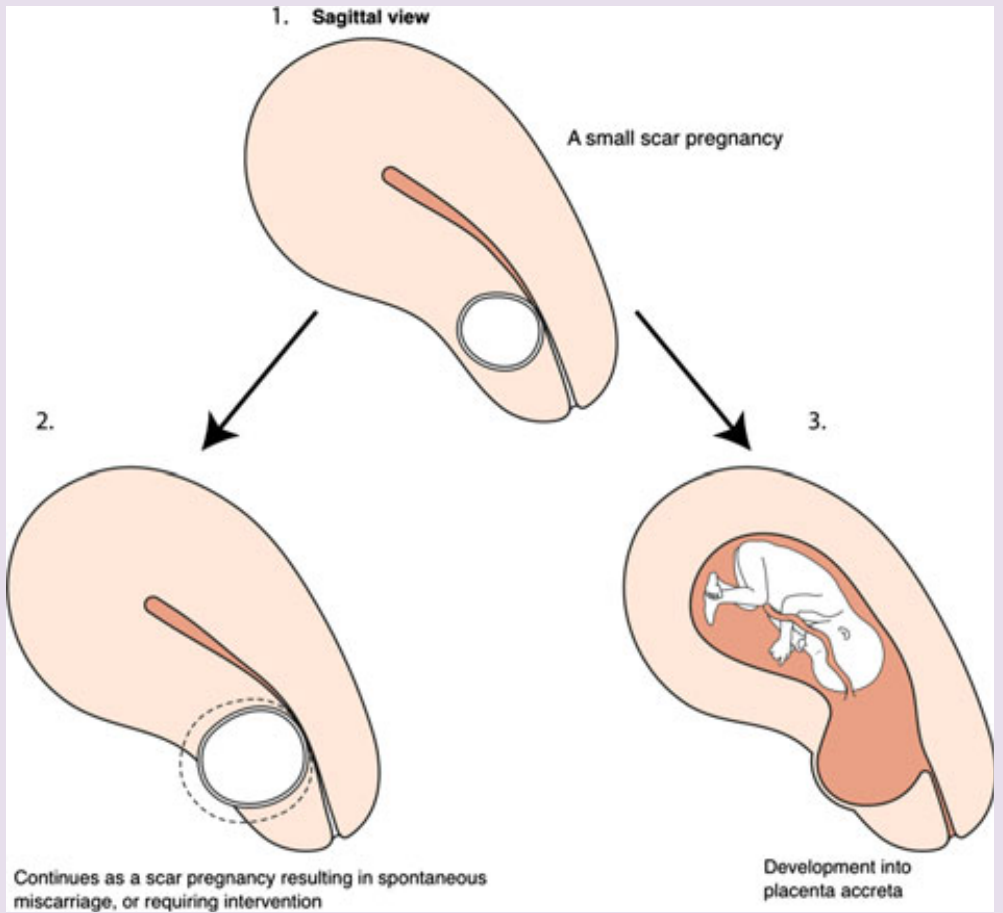

Figure 1. The fate of a low pregnancy sac in a scarred uterus. 
In this hypothesis, we propose that all women who have had previous caesarean sections should have a transvaginal scan examination between 6 and 8 weeks of gestation in any subsequent pregnancy. The purpose of this scan would be to identify the implantation site of the gestational sac. If the scan showed that the sac was implanted in the fundus, this should rule out the possibility of a scar pregnancy and the potential of placenta accreta in a woman with a previous scar. The other possibility is that of a low or isthmic gestational sac. This has a higher chance of adverse events, including miscarriage, scar pregnancy and placenta accreta. Women with a low gestational sac would be categorised as higher risk and require further evaluation and monitoring.

We think it possible to screen based on fundal versus non-fundal or low pregnancy implantation, particularly when the gestational sac is small. Visualisation of the small, early gestational sac in relation to the uterine fundus could develop into a simple screening test (see Figure 1). As the gestational sac becomes larger it fills the whole cavity and it encroaches on the niche of the previous caesarean section scar. Identification of the original implantation site will therefore become harder.

A screening programme to identify the location of the gestational sac at 6-8 weeks of gestation would be simple and could provide both women and obstetricians with the valuable knowledge of not having a risk of placenta accreta if the gestational sac was fundal.

We believe that minimal changes in training would be required. Research will provide women and clinicians with therapeutic options, including termination of pregnancy if a scar, isthmic or low gestational sac was shown to be firmly associated with placenta accreta. Research should be funded and encouraged to clarify the poorly understood natural history of caesarean section scar pregnancies. It is not known what proportion (if not all) of low implantations result in caesarean section scar pregnancies/placenta accreta. Without this information, one runs a possible risk of suggesting termination for a pregnancy that may never have developed into accreta. Termination of a pregnancy implanted in the scar is not without risks. Implementation is possible only after the natural history of placenta accreta is understood and after trials have assessed the benefits, risks and cost of such a screening programme.

\section{Disclosure of interests}

All authors declare no conflicts of interest.

H El-Refaey, ${ }^{\text {a }}$ F El-Hibri' ${ }^{b}$ \& N Youakim ${ }^{c}$

${ }^{a}$ Department of Obstetrics and Gynaecology, Chelsea and Westminster Hospital, London, UK

${ }^{b}$ Royal Surrey County Hospital, Guildford, UK

${ }^{c}$ University Hospital, Coventry, UK 